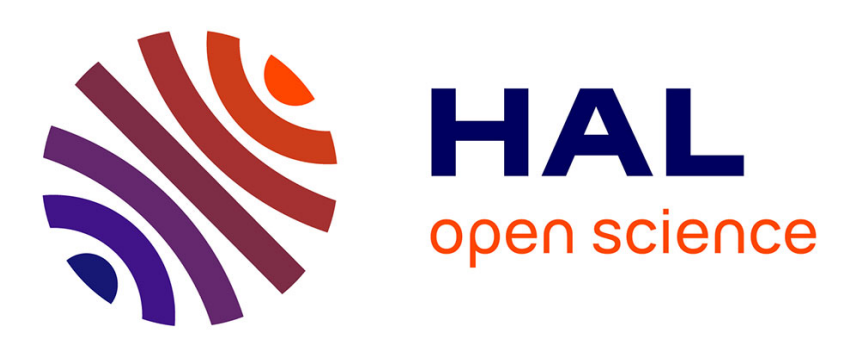

\title{
Unbiased observer synthesis using dynamic output allocation for discrete-time linear plants with redundant sensors
}

\author{
Andrea Cristofaro, Luca Zaccarian
}

\section{- To cite this version:}

Andrea Cristofaro, Luca Zaccarian. Unbiased observer synthesis using dynamic output allocation for discrete-time linear plants with redundant sensors. 2017 IEEE 56th Annual Conference on Decision and Control (CDC), Dec 2017, Melbourne, Australia. 10.1109/CDC.2017.8263902 . hal-01700158

\section{HAL Id: hal-01700158 \\ https://hal.laas.fr/hal-01700158}

Submitted on 17 Feb 2018

HAL is a multi-disciplinary open access archive for the deposit and dissemination of scientific research documents, whether they are published or not. The documents may come from teaching and research institutions in France or abroad, or from public or private research centers.
L'archive ouverte pluridisciplinaire HAL, est destinée au dépôt et à la diffusion de documents scientifiques de niveau recherche, publiés ou non, émanant des établissements d'enseignement et de recherche français ou étrangers, des laboratoires publics ou privés. 


\title{
Unbiased observer synthesis using dynamic output allocation for discrete-time linear plants with redundant sensors
}

\author{
Andrea Cristofaro and Luca Zaccarian
}

\begin{abstract}
The problem of output redundancy in discrete-time linear plants is addressed, wherein the presence of redundant sensors is motivated by unknown bias or faults affecting the measurement equation. In this context, we focus on the design of a nonlinear estimation procedure consisting in a bias estimator together with a linear Luenberger structure augmented with an adaptive weighted pseudo-inverse combination of the available measurements. It is shown that the proposed scheme is characterized by higher performances compared to the classical compensation of the observer output injection using the estimated bias. Simulation results are given to illustrate the potential behind the proposed solution.
\end{abstract}

\section{INTRODUCTION}

Input redundancy has been widely investigated, to a large extent in the framework of control allocation [1], [2], which is a modular setup where the properties of a redundant set of inputs are exploited in order to state a constrained optimization scheme that incorporates both primary and secondary control objectives. Conversely, output allocation, or dual redundancy, is a less studied problem yet very interesting and many-sided. The Kalman filter and the extended Kalman filter [3], being estimation methods aiming at the minimization of the covariance error, naturally benefit from the presence of a redundant set of outputs. However, such redundancy is usually not explicitly analyzed and is used in a passive way. As for the case of actuator redundancy, the presence of redundant outputs might be very helpful in handling sensor faults and biased measurements. In this regard, some results pertaining safety and reliability of marine systems have been proposed [4]. On the other hand, sensor fault detection is a challenging problem, as there are no unquestionable methods to establish whether a sensor is faulty or not, based on the measurement value. Several approaches have been proposed [5], such as robust observers [6], [7], consensus based schemes [8], adaptive approximation [9] or statistical methods [10]. Robust observers are typically designed for systems with structured faults by exploiting geometric properties to achieve fault decoupling. The underlying idea of consensus methods is to put more trust in the measurements on which the largest group of sensors agree, while adaptive approximation consists in the online reconstruction of a fault estimate, typically by means of neural networks architectures.

The method presented in this paper arises instead from the elementary yet fundamental observation that, among all the possible combinations of outputs, there is one which is less sensitive or even insensitive to a given perturbation. On the other hand, as the perturbing signal is typically unknown and unpredictable, such an optimal combination is also generally unknown. In the absence of faults and external perturbations, an equivalent full-rank, lower dimensional output model can be extracted using a weighted pseudo-inverse, where the weights indicate the burden on each individual sensor. The scope of the paper is to propose an adaptation scheme for such weights, with the aim of defining dynamic pseudo-inverses being able to asymptotically converge to an optimal combination of outputs, where optimality has to be interpreted as the minimization of the perturbation effect in the output estimation error of a given state observer. In other words, the introduction of dynamic pseudo-inverses allows

A. Cristofaro is with School of Science and Technology, University of Camerino, Italy. email: andrea.cristofaro@unicam.it L. Zaccarian is with LAAS-CNRS, Université de Toulouse, CNRS, Toulouse (France) and Dip. di Ingegneria Industriale, University of Trento (Italy).

This work is supported by the Research Council of Norway through the Centres of Excellence funding scheme, project No. 223254 AMOS, and by grant PowerLyap funded by CaRiTRo. 
to design a fault-tolerant adaptive observer with the nice property of being self-tuning. Moreover, the asymptotic value of the adaptive weights is a clear indication of faults or biases presence, location and relative magnitude. A similar paradigm has been introduced in [11] for continuous-time systems, and the extension to discrete-time systems is presented here. We point out that this is not a mere revisiting of the previous results in the discrete-time framework, since a completely different design procedure is proposed here. In particular, the synthesis of two observers is proposed, to be used in cascade: the first one is responsible of estimating the bias, while the second one reconstructs the state of the system by selecting the best combination of outputs based on the estimated bias.

The paper is structured in five main sections. In Section II the problem is formally stated, and the design of the bias estimator is addressed in Section III. A general architecture for the unbiased state estimator using dynamic output allocation is exploited in Section IV, while a specific algorithm to compute the adaptation scheme is proposed in Section V. Finally, in Section VI some numerical examples illustrate the method performances and validate the theoretical results.

\section{PROBLEM DEFINITION}

Let us consider the linear time-invariant discrete-time plant

$$
\begin{aligned}
x_{k+1} & =A x_{k}+B u_{k} \\
y_{k} & =C x_{k}+\varphi,
\end{aligned}
$$

where $x \in \mathbb{R}^{n}, u \in \mathbb{R}^{m}$ and $y \in \mathbb{R}^{p}$ are state, input and output of the plant, respectively, $A, B, C$ are matrices of appropriate dimensions and $\varphi$ is an unknown but constant bias affecting the outputs of the system.

For plant (1) we assume that the output $y$ is redundant, in the sense that some measurements are linear combination of the other ones. We also assume that the state of the plant is detectable from $y$ (otherwise no state estimation could be possible). Finally, we assume that the constant bias $\varphi$ can be detected from output $y$, which corresponds to requiring that matrix $A$ cannot generate constant responses (namely it has no eigenvalues in 1). These assumptions are formalized below.

Assumption 1: Plant (1) is such that the following holds:

1) Output Redundancy. The output is redundant namely there exists an integer $q<p$ and matrices $Y \in \mathbb{R}^{p \times q}, H \in \mathbb{R}^{q \times n}$ such that $q:=\operatorname{rank}(C)=\operatorname{rank}(H)$, and $C=Y H$.

2) Detectability of $x$. Pair $(C, A)$ is detectable (therefore also $(H, A)$ is detectable);

3) Detectability of $\varphi$. Matrix $A-I$ is nonsingular (namely, matrix $A$ has no eigenvalue in 1 ).

While the first property (redundancy) characterizes the peculiar feature exploited in the scheme proposed in this paper, and is not necessary for estimating $x$ and $\varphi$, we emphasize that the two subsequent items in Assumption 1 are indeed necessary to build a finite-time or asymptotic observer of $x$ in the presence of the unknown constant bias $\varphi$. This is easily seen by augmenting plant (1) with an exosystem and realizing that item 3 is a necessary condition for detectability.

Regarding item 1, as long as $q<p$, there exists an infinite number of pairs $(Y, H)$ with $C=Y H$. Having selected one, we refer to such $H$ as an extracted full rank (EFR) output matrix. For any selection of that matrix, since we assume detectability in Assumption 1, in the absence of the bias $\varphi$, a full order Luenberger observer can be designed:

$$
\hat{x}_{k+1}=A \hat{x}_{k}+B u_{k}+L\left(H x_{k}-H \hat{x}_{k}\right)
$$

where $L$ is a suitable output error injection gain ensuring that $\left|x_{k}-\hat{x}_{k}\right| \rightarrow 0$ as $k$ tends to $\infty$.

The scope of this paper is to develop a design procedure for an unbiased observer, i.e. an observer that, using the injection of the redundant output $y$, guarantees the same performance as the nominal observer (2), despite the presence of the perturbation $\varphi$. 


\section{AsYMPTOTIC BIAS ESTIMATION}

As a first ingredient of our scheme, we exploit Assumption 1 to design an asymptotic estimate $\hat{\varphi}$ of the bias $\varphi$. To this end, we first observe that $\varphi$ always admits a decomposition of the following form:

$$
\varphi=Y \varphi_{Y}+\varphi_{o}
$$

with $\varphi_{Y} \in \mathbb{R}^{p}$ and where $\varphi_{o} \in[\operatorname{span}(Y)]^{\perp}$ satisfies $Y^{T} \varphi_{o}=0$. Vector $\varphi_{o}$ can be readily extracted from the output by projecting measurement $y_{k}$ onto $[\operatorname{span}(Y)]^{\perp}$ at any time $k \in \mathbb{Z}_{\geq 0}$, as follows: $\varphi_{o}=\mathbb{P}_{Y \perp} y_{k}:=\left(I-\mathbb{P}_{Y}\right) y_{k}$, where we used the projection matrix $\mathbb{P}_{Y}:=Y\left(Y^{T} Y\right)^{-1} Y^{T}$.

Thanks to item 2 of Assumption 1, we can select a Luenberger gain $M$ such that $A-M H$ is a Schur matrix (namely its eigenvalues all have magnitude smaller than one). Using this output injection gain, we introduce an observer with the scope of estimating $\varphi_{Y}$ :

$$
\xi_{k+1}=A \xi_{k}+B u_{k}+M \mathbb{P}_{Y}\left(y_{k}-C \xi_{k}\right) .
$$

The asymptotic estimate $\hat{\varphi}$ of $\varphi$ can be then determined as follows:

$$
\begin{aligned}
\eta_{k} & :=\mathbb{P}_{Y} y_{k}-H \xi_{k} \\
\hat{\varphi}_{k} & :=Y\left(I+H(I-A)^{-1} M\right) \eta_{k}+\mathbb{P}_{Y^{\perp}} y_{k},
\end{aligned}
$$

where the output gain in (4c) is well defined due to item 3 of Assumption 1. The effectiveness of (4) at estimating $\varphi$ is established in the next proposition.

Proposition 1: If matrix $A-M H$ is Schur, then the difference $\hat{\varphi}_{k}-\varphi$ converges exponentially to zero. In particular, setting $\epsilon_{k}:=x_{k}-\xi_{k}$, the error dynamics reads as:

$$
\epsilon_{k+1}=(\underbrace{A-M H}_{:=A_{c l}}) \epsilon_{k}-M \varphi_{Y} .
$$

Example 1: An unbiased observer can be readily designed by injecting directly the bias estimate $\hat{\varphi}_{k}$, e.g. using an output injection of the form $y_{k}-\hat{\varphi}_{k}$ into (2). However, as it will be highlighted in the present example, the resulting estimation is far from being accurate in the presence of noisy measurements or time-varying biases.

Consider the following plant:

$$
\begin{gathered}
A=\left[\begin{array}{rrr}
1 & 0 & 1 \\
3 & 9 & 1 \\
1 & -1 & 3
\end{array}\right], \quad B=\left[\begin{array}{r}
1 \\
5 \\
-1
\end{array}\right], \\
C=\left[\begin{array}{lll}
1 & 1 & 0 \\
1 & 1 & 0
\end{array}\right], \quad Y=\left[\begin{array}{l}
1 \\
1
\end{array}\right], \quad H=\left[\begin{array}{lll}
1 & 1 & 0
\end{array}\right] .
\end{gathered}
$$

Referring to (2) and (4a), the gains of the observers $\hat{x}_{k}$ and $\xi_{k}$ are chosen as

$$
M=\left[\begin{array}{r}
31.8996 \\
-18.9026 \\
86.3395
\end{array}\right], \quad L=\left[\begin{array}{r}
27.3860 \\
-14.9860 \\
73.8200
\end{array}\right]
$$

with $\operatorname{spec}(A-M H)=\{0\}$ and $\operatorname{spec}(A-L H)=\{0.1,0.2,0.3\}$. We notice that the considered plant is unstable. i.e. $\operatorname{spec}(A)=\{8.7534,0.4861,3.7605\}$ : accordingly, a stabilizing feedback controller $u=-K \hat{x}$ is designed, with gain $K=\left[\begin{array}{lll}-1.0345 & 1.8357 & -3.0558\end{array}\right]$ and closed-loop poles $\operatorname{spec}(A-$ $B K)=\{0.5,0.6,0.7\}$.

A constant bias $\varphi$ is assumed to affect the output equation, with

$$
\varphi=\left[\begin{array}{l}
1 \\
2
\end{array}\right], \quad y_{k}=C x_{k}+\varphi
$$


As clearly illustrated in Figure III, the estimation of the bias is fairly accurate in the absence of sensor noise and provides a fast decay of the estimation error $|e|=|x-\hat{x}|$. However, when a perturbed bias is considered, i.e.

$$
\varphi_{k}=(1+0.5 w(k)) \varphi
$$

where $w(k)$ is a white noise process, a remarkable deterioration of performances occurs and is visible at the top of Figure III. This is also strongly reflected in a poor estimation accuracy as shown at the bottom of Figure III.
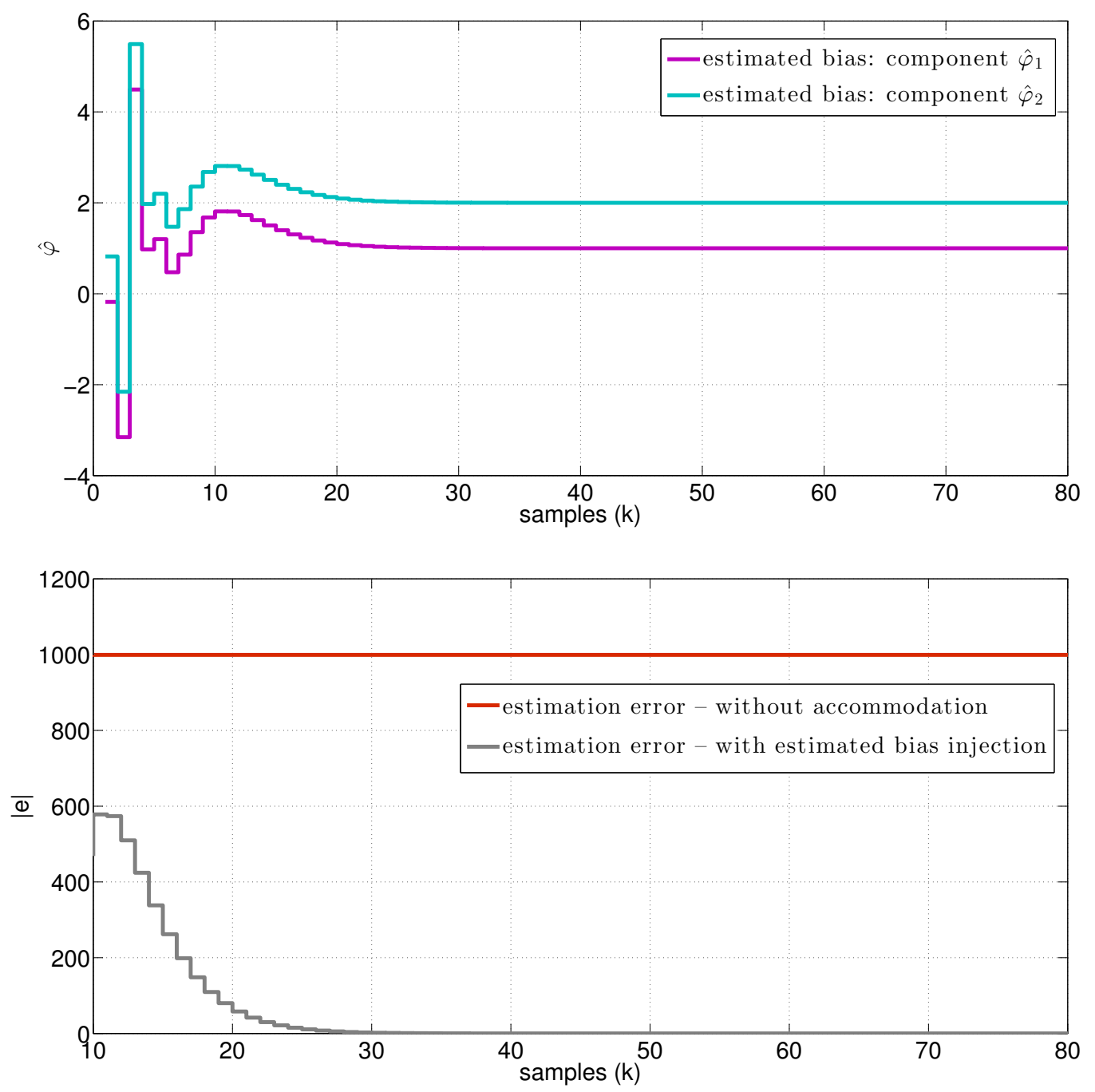

Fig. 1. Example 1: Bias estimation (top) and state estimation error (bottom) without measurement noise.

\section{AN OBSERVER USING DYNAMIC PSEUDOINVERSES}

Motivated by the drawback highlighted in the latter example, in this section we investigate the design of enhanced observers by using the estimation of bias and simultaneously exploiting the sensor redundancy with the aim of reducing the effect of noisy measurements and bias oscillation on the estimation performance. 

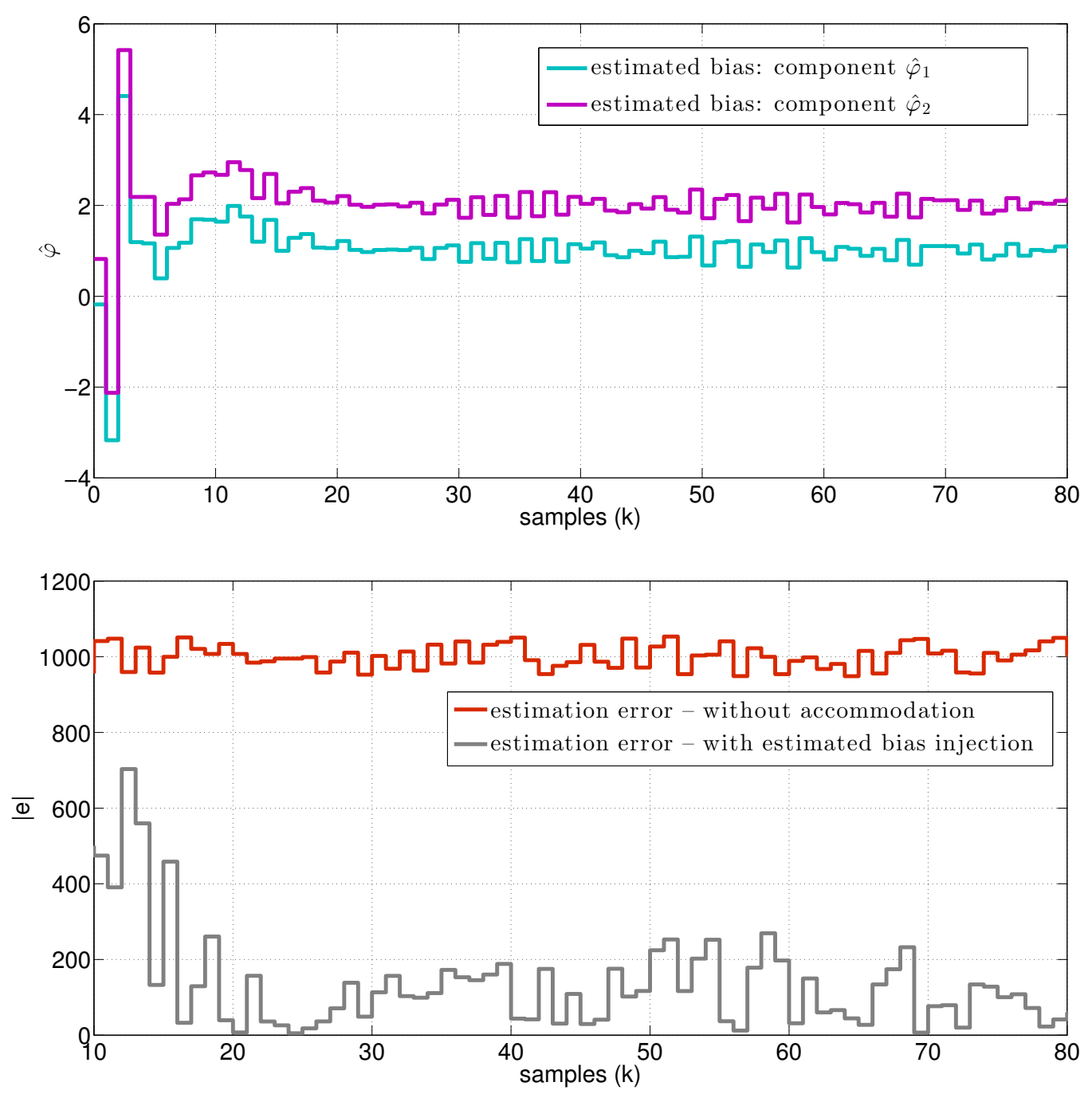

Fig. 2. Example 1: Bias estimation (top) and state estimation error (bottom) with measurement noise.

\section{A. Observer architecture and its properties}

Similar to (2), given any gain matrix $L$ such that $A-L H$ is Schur, we construct the following asymptotic observer:

$$
\begin{aligned}
\hat{x}_{k+1} & =A \hat{x}_{k}+B u_{k}+L \Pi\left(z_{k}\right)\left(y_{k}-\hat{y}_{k}\right) \\
\hat{y}_{k} & =C \hat{x}_{k} \\
z_{k+1} & \in G\left(z_{k}, \hat{\varphi}_{k}\right),
\end{aligned}
$$

where the additional state $z \in \mathbb{R}^{q}$ is selected to perform an on-line adaptation of matrix function $G$, which corresponds to the weighted pseudoinverse

$$
\Pi(z):=R(z)^{-1} \Gamma(z):=(\underbrace{Y^{T} \operatorname{diag}(z) Y}_{=: R(z)})^{-1} \underbrace{Y^{T} \operatorname{diag}(z)}_{=: \Gamma(z)} .
$$

The set-valued mapping $G$ is a suitable ingredient of the design procedure, specified below. The goal in the design of $G$ is to ensure that $z$ automatically selects the "best" information content among the redundant measurements $y_{k}$ through $\Pi\left(z_{k}\right)$. To this end, we first observe that $\Pi\left(z_{k}\right)$ is scale invariant, i.e. $\Pi\left(z_{k}\right)=\Pi\left(\mu z_{k}\right)$ for any $\mu \neq 0$, and for this reason it is helpful to restrict the vector $z_{k}$ to lie on the 
unit sphere $\mathbb{S}^{p-1}$. Moreover, we observe that map $\Pi$ in (9) is only well defined if matrix $R$ is invertible. Due to this fact, we only focus on values of $z$ evolving in the following set:

$$
\mathcal{Z}_{\epsilon}:=\left\{z \in \mathbb{S}^{p-1}:|\operatorname{det}(R(z))| \geq \epsilon\right\}
$$

which is compact because it is the intersection between the compact set $\mathbb{S}^{p-1}$ and a closed unbounded set.

We observe that for any pair $z, v \in \mathbb{R}^{p}$ the following identity holds $\operatorname{diag}(z) v=\operatorname{diag}(v) z$, and therefore

$$
\Gamma(z) \varphi=\Gamma(\varphi) z
$$

where $\Gamma$ has been defined in (9). By developing the output injection term in (8), we may appreciate the importance of the term appearing in (8). Indeed, we get, also using (11),

$$
\begin{aligned}
& L \Pi\left(z_{k}\right)\left(y_{k}-\hat{y}_{k}\right)=L R(z)^{-1} \Gamma(z)(Y H x+\varphi-Y H \hat{x}) \\
& \quad=L R(z)^{-1} \Gamma(z) Y H(x-\hat{x})+L R(z)^{-1} \Gamma(z) \varphi \\
& \quad=L(H x-H \hat{x})+L R(z)^{-1} \Gamma(\varphi) z,
\end{aligned}
$$

where we used $R(z)^{-1} \Gamma(z) Y=R(z)^{-1} Y^{T} \operatorname{diag}(z) Y=I$.

Combining equation (12) with observer (8) and plant (1), we can define the estimation error $e:=x-\hat{x}$, whose dynamics can be written as:

$$
\begin{aligned}
& e_{k+1}=(A-L H) e_{k}+L R\left(z_{k}\right)^{-1} \Gamma(\varphi) z_{k}, \\
& z_{k+1} \in G\left(z_{k}, \hat{\varphi}_{k}\right),
\end{aligned}
$$

showing a convenient structure only involving states $e$ and $z$, and input $\hat{\varphi}$.

The error dynamics (13) illustrates the effectiveness of the proposed solution in terms of providing an observer that, in the presence of the unknown bias $\varphi$, structurally recovers the estimation error transient experienced with unbiased dynamics (2). Such a recovery is however only possible if the perturbation term $R(z)^{-1} \Gamma(z) \varphi$ becomes zero, at least asymptotically. This goal motivates the following property that we state for the set-valued function $G$ and that will be guaranteed by the construction proposed later in Section V.

Property 1: Given the constant bias $\varphi$ in (1), there exists a scalar $\epsilon>0$ such that the set-valued map $G$ is nonempty and locally bounded and outer semi-continuous relative to $\mathcal{Z}_{\epsilon} \times \mathbb{R}^{p}$. Moreover, for each $z \in \mathcal{Z}_{\epsilon}, G(z, \varphi) \subset \mathcal{Z}_{\epsilon}$ and

$$
z^{+} \in G(z, \varphi) \Rightarrow\left(\Gamma(\varphi) z^{+}=0\right) \text { or }\left(\left|\Gamma(\varphi) z^{+}\right|<|\Gamma(\varphi) z|\right) .
$$

Property 1 ensures that, as long as $G$ is fed with the correct bias signal $\varphi$, it produces a set of possible selections for the next value of $z$, that leads to an improvement of the filtering action in (12), unless its value is already zero. Based on Property 1, we can now state the following result, that is a second important baseline result for the proposed scheme.

Proposition 2: Consider plant (1) observer (8) driven by the constant input $\hat{\varphi}_{k}=\varphi$ for all $k$. If function $G$ is outer semi-continuous and satisfies Property 1 for a suitable $\epsilon>0$, then for any $L$ ensuring that $A-L C$ is Schur, the compact set $\mathcal{A}_{2}:=\{(e, z): \Gamma(\varphi) z=0, e=0\}$ is globally asymptotically stable for the ensuing error dynamics (13).

Our main result below follows from Propositions 1 and 2 after making the key observation that $\varepsilon_{k}$ converges exponentially to the unique equilibrium:

$$
\varepsilon^{*}:=-(I-(A-M H))^{-1} M \varphi_{Y} .
$$

Based on the above derivations, our goal is to focus on the following compact set:

$$
\mathcal{A}:=\left\{(\varepsilon, e, z): \Gamma(\varphi) z=0, \varepsilon=\varepsilon^{*}, e=0\right\}
$$


whose desirable stability properties are established in the main result below, whose proof is omitted due to space constraints and is based on a discrete-time version of classical reduction theorems [12].

Theorem 1: Consider any pair of gains $M$ and $L$ such that $A-L H$ and $A-M H$ are Schur. If function $G$ is outer semi-continuous and satisfies Property 1 for a suitable $\epsilon>0$, then the compact set (15) is globally asymptotically stable for the ensuing error dynamics (5), (13).

\section{B. Scale invariance and low-pass filtering}

As already mentioned, the weighted pseudoinverse $\Pi(z)$ is invariant under scaling of $z$. On the other hand, a quasi-invariance property with respect to the bias $\varphi$ is also guaranteed. Indeed if $\Pi(z)$ is the optimal pseudoinverse for $\varphi$, i.e. $\Pi(z) \varphi=0$, then $\Pi(z)$ is optimal for the whole space $\operatorname{span}(\varphi)$. Such a feature of the output allocation based observer (8) is a further advantage compared to the injection corrected observer discussed in Example 1 in terms of transient performances.

To illustrate this fact, let us consider a time-varying perturbation term $\varphi_{k}$ with $\varphi_{k}=g_{k} \varphi, g_{k} \in \mathbb{R} \backslash$ $\{0\} \forall k \in \mathbb{N}$. Due to the presence of the time-varying factor $g_{k}$, the auxiliary observer $\xi_{k}$ is no longer capable to ensure convergence of $\hat{\varphi}_{k}$ to $\varphi$. Moreover, since the gain $M$ of a fast observer is typically large, some overshoots are likely to arise in the estimated bias $\hat{\varphi}_{k}$, with a potential inaccuracy of the observer $\hat{x}$ and the associated output allocator. However, this problem might be overcome by introducing a filter that processes the estimate $\hat{\varphi}_{k}$ before this signal is fed to the observer (8) and the output allocator.

To this end, we propose an operative procedure with the aim of reducing the effect of high frequency components of the factor $g_{k}$ on the computation of the optimal weights $z_{k}$.

Algorithm 1 (Scaling \& Filtering):

i) Project $\hat{\varphi}_{k}$ onto the homogeneous $\operatorname{space}^{1} \hat{\mathbb{R}}^{p}=\left\{\zeta \in \mathbb{R}^{p}: \zeta_{p}=1\right\}$ :

$$
\hat{\varphi}_{k} \Rightarrow \hat{\varphi}_{k}^{*}:=\mathbb{P}_{\hat{\mathbb{R}}^{p}} \hat{\varphi}_{k}=\hat{\varphi}_{k} / \hat{\varphi}_{k, p}
$$

ii) Low-pass filter the signal $\hat{\varphi}_{k}^{*}$ :

$$
\hat{\varphi}_{k}^{* *}=(1-\tau) \hat{\varphi}_{k-1}^{* *}+\tau \hat{\varphi}_{k}^{*}
$$

where $\tau$ is a suitable filter parameter.

iii) Feed the processed signal $\hat{\varphi}_{k}^{* *}$ to the output allocator in (8):

$$
z_{k+1} \in G\left(z_{k}, \hat{\varphi}_{k}^{* *}\right) .
$$

\section{A possible SELECTION OF THE MAP $G$}

An algorithm for generating a map $G$ satisfying Property 1 is proposed. The underlying idea is to evaluate the norm of $\Gamma(\varphi) z$ along rank-one increments. In fact, denoting by $y_{(i)}$ the columns of $Y^{T}$, the matrix $R(z)=Y^{T} \Gamma(z) Y$ can be written as a finite sum of rank-one matrices:

$$
R(z)=\sum_{i=1}^{p} z_{i} \Upsilon_{i}, \quad \Upsilon_{i}=y_{(i)} y_{(i)}^{T} .
$$

Let us denote by $\left\{\mathbf{e}_{i}\right\}, i=1, \ldots, p$, the vectors of the canonical basis of $\mathbb{R}^{p}$. Let $\bar{z}$ be such that $\operatorname{det}(R(\bar{z})) \neq 0$, and consider the rank-one perturbation $R\left(\bar{z}+\zeta \mathbf{e}_{i}\right)$. By the Sherman-Morrison formula [13] one has

$$
R^{-1}\left(\bar{z}+\zeta \mathbf{e}_{i}\right)=R^{-1}(\bar{z})-\zeta \frac{R^{-1}(\bar{z}) \Upsilon_{i} R^{-1}(\bar{z})}{1+\zeta y_{(i)}^{T} R^{-1}(\bar{z}) y_{(i)}},
$$

\footnotetext{
${ }^{1}$ It is assumed without loss of generality that $\hat{\varphi}_{k, p} \neq 0$. The choice of rescaling the last component is arbitrary, any other non zero component can be used.
} 
that is clearly well-defined if and only if

$$
1+\zeta y_{(i)}^{T} R^{-1}(\bar{z}) y_{(i)} \neq 0
$$

For $\epsilon>0$, the $(\epsilon, \bar{z}, i)$-feasible set $\Lambda(\epsilon, \bar{z}, i)$ is defined as

$$
\Lambda(\epsilon, \bar{z}, i)=\left\{\zeta \in \mathbb{R}:\left|1+\left(\zeta-\bar{z}_{i}\right) y_{(i)}^{T} R^{-1}(\bar{z}) y_{(i)}\right| \geq \epsilon\right\}
$$

More precisely, the decomposition $\Lambda(\epsilon, \bar{z}, i)=\Lambda_{+}(\epsilon, \bar{z}, i) \cup \Lambda_{-}(\epsilon, \bar{z}, i)$ holds with

$$
\begin{gathered}
\Lambda_{+}(\epsilon, \bar{z}, i):=\left\{\zeta \in \mathbb{R}: 1+\left(\zeta-\bar{z}_{i}\right) y_{(i)}^{T} R^{-1}(\bar{z}) y_{(i)} \geq \epsilon\right\} \\
\Lambda_{-}(\epsilon, \bar{z}, i):=\left\{\zeta \in \mathbb{R}: 1+\left(\zeta-\bar{z}_{i}\right) y_{(i)}^{T} R^{-1}(\bar{z}) y_{(i)} \leq-\epsilon\right\} .
\end{gathered}
$$

As a matter of fact, as $i$ varies in $\{1, \ldots, p\}$, the above objects form a family of $2 p$ disjoint closed sets. Set $S(\varphi):=\Gamma(\varphi) Y Y^{T} \Gamma(\varphi)$, whose entries are denoted by $\left\{s_{i j}\right\}_{i, j=1}^{p}$, and consider the family of minimization problems

$$
\min _{z \in p} F(z)=\min _{z \in p} \frac{z^{T} S(\varphi) z}{z^{T} z}
$$

subject to

$$
z=\bar{z}+\zeta \mathbf{e}_{\mathbf{i}}, \quad \zeta \in \Lambda(\epsilon, \bar{z}, i)
$$

Notice that the minimization problem (17) is equivalent to minimizing function $|\Gamma(\varphi) z|^{2}$ with $z$ restricted to the projection on the unit sphere $\mathbb{S}^{p-1}$ of the line generated by $\bar{z}+\zeta \mathbf{e}_{\mathbf{i}}$ for all $\zeta \in \Lambda(\epsilon, \bar{z}, i)$. The advantage of only focusing on a line search is that one can exploit the structure of the disconnected set $\Lambda$ in (16) and perform the ensuing one-dimensional optimization by evaluting increments $z_{i}$ along the direction $\mathbf{e}_{i}$. By defining the projection operator $E_{i}:=I-\mathbf{e}_{i} \mathbf{e}_{i}^{T}$, the function $F$ in optimization (17) can be rewritten as the scalar rational map

$$
\begin{aligned}
F_{i}\left(z_{i}\right) & :=F\left(E_{i} \bar{z}+z_{i} \mathbf{e}_{i}\right) \\
& =\frac{s_{i i} z_{i}^{2}+2 z_{i} \sum_{j \neq i} s_{i j} \bar{z}_{j}+\sum_{j, \ell \neq i} s_{j \ell} \bar{z}_{j} \bar{z}_{\ell}}{z_{i}^{2}+\sum_{j \neq i} \bar{z}_{j}^{2}},
\end{aligned}
$$

or, more concisely, as $F_{i}\left(z_{i}\right)=\frac{c_{2 i} z_{i}^{2}+c_{1 i} z_{i}+c_{0 i}}{z_{i}^{2}+a_{0 i}}$ where $c_{2 i}=s_{i i}, \quad c_{1 i}=2 \sum_{j \neq i} s_{i j} \bar{z}_{j}, \quad c_{0 i}=\sum_{j, \ell \neq i} s_{j \ell} \bar{z}_{j} \bar{z}_{\ell}$, and $a_{0 i}=\sum_{j \neq i} \bar{z}_{j}^{2}$. Computing the first derivative yields

$$
F_{i}^{\prime}\left(z_{i}\right)=\frac{-c_{1 i} z_{i}^{2}+2 z_{i}\left(c_{2 i} a_{0 i}-c_{0 i}\right)+c_{1 i} a_{0 i}}{\left(z_{i}^{2}+a_{0 i}\right)^{2}} .
$$

Noticing that $\left(c_{2 i} a_{0 i}-c_{0 i}\right)^{2}+c_{1 i}^{2} a_{0 i} \geq 0$, the equation $F_{i}^{\prime}(\delta)=0$ always admits two real solutions

$$
z_{i_{ \pm}}^{\circ}=\frac{-\left(c_{2 i} a_{0 i}-c_{0 i}\right) \pm \sqrt{\left(c_{2 i} a_{0 i}-c_{0 i}\right)^{2}+c_{1 i}^{2} a_{0 i}}}{-c_{1 i}}
$$

provided that $c_{1 i} \neq 0$. Moreover, it can be verified that the minimum is always achieved in $z_{i_{-}}^{\circ}$. Whether such solution is still consistent in the degenerate scenario $c_{1 i}=0$ has to be addressed. It can be verified computing the limit $\lim _{c_{1 i} \rightarrow 0} z_{i_{-}}^{\circ}$ that the condition $\left(c_{2 i} a_{0 i}-c_{0 i}\right)<0$ is necessary for feasibility to be preserved when $c_{1 i}=0$. On the other hand, exploiting the expression of these coefficients, the latter inequality appears unlikely to be satisfied. To overcome such an issue, one might introduce artificial restrictions on the admissible set of increments, e.g. allowing $z_{i}$ to vary in a compact set only. In this regard, for a fixed $K>0$ let us consider the sliding intervals $\mathcal{I}(K, \bar{z}, i):=\left[-K\left|\bar{z}_{i}\right|, K\left|\bar{z}_{i}\right|\right]$ and, accordingly, set

$$
\Lambda_{ \pm}(K, \epsilon, \bar{z}, i):=\Lambda_{ \pm}(\epsilon, \bar{z}, i) \cap \mathcal{I}(K, \bar{z}, i)
$$


The boundary of this constrained feasible set is given by the collection of four singletons:

$$
\partial \Lambda_{ \pm}(K, \epsilon, \bar{z}, i)=\left\{K\left|\bar{z}_{i}\right|,-K\left|\bar{z}_{i}\right|, \delta_{+}(\epsilon, \bar{z}, i), \delta_{-}(\epsilon, \bar{z}, i)\right\}
$$

where $\delta_{ \pm}(\epsilon, \bar{z}, i):=\frac{-1+\bar{z}_{i} y_{(i)}^{T} R^{-1}(\bar{z}) y_{(i)} \pm \epsilon}{y_{(i)}^{T} R^{-1}(\bar{z}) y_{(i)}}$.

Based on the computations above it can be inferred that, for any fixed $i$, the constrained minimum is achieved either in $z_{i_{-}}^{\circ}$ if the latter point lies inside the feasible set $\Lambda_{+}(K, \epsilon, \bar{z}, i) \cup \Lambda_{-}(K, \epsilon, \bar{z}, i)$, or in one of the boundary points (19) otherwise. Evaluating such minimum and comparing the results as $i$ varies in $\{1, \ldots, p\}$, the preferred direction of increment $z_{i}$ for minimizing the norm of $\Gamma(\varphi) z$ can be identified by a finite number of operations: such a direction is then used to assign the update map $z^{+}$satisfying Property 1. Summarizing, we can state the following algorithm and the subsequent proposition.

Algorithm 2: 응 Update algorithm for $z$

Input: $\bar{z}, \varphi$

$i=1$

While $i \leq p$

If $c_{1 i} \neq 0 \& z_{i_{-}}^{\circ} \in \Lambda_{ \pm}(K, \epsilon, \bar{z}, i)$

then $z_{\min }(i)=z_{i_{-}}^{\circ}$

else $z_{\min }(i)=\arg \min \left\{\begin{array}{c}F_{i}\left(K\left|\bar{z}_{i}\right|\right), F_{i}\left(-K\left|\bar{z}_{i}\right|\right), \\ F_{i}\left(\delta_{+}(\epsilon, \bar{z}, i)\right), F_{i}\left(\delta_{-}(\epsilon, \bar{z}, i)\right)\end{array}\right\}$

$i=i+1$

end

$i^{\star}=\arg \min _{i=1, \ldots, p}\left\{F_{i}\left(z_{\min }(i)\right)\right\}$

$\zeta^{\star}=z_{\min }\left(i^{\star}\right)$

Output: $i^{\star}, \zeta^{\star}$

The next proposition establishes desirable properties of Algorithm 2 to the end of satisfying Property 1.

Proposition 3: Consider the following definition of the update map $G$ :

$$
z^{+}=\frac{z+\left(\zeta^{\star}-z_{i^{\star}}\right) \mathbf{e}_{i^{\star}}}{\left|z+\left(\zeta^{\star}-z_{i^{\star}}\right) \mathbf{e}_{i^{\star}}\right|}
$$

where $i^{\star}$ and $\zeta^{\star}$ are provided by Algorithm 2 with input $z$.

Then the sequence of weights $z_{k}$ satisfies for any $k \in \mathbb{N}$ the inequality $\left|\Gamma(\varphi) z_{k+1}\right| \leq\left|\Gamma(\varphi) z_{k}\right|$ together with the feasibility condition $\operatorname{det}\left(R\left(z_{k}\right)\right) \neq 0$.

Remark 1: It is worth noticing that, implementing Algorithm 2 and using the recipe proposed in Proposition 3, Property 1 is fulfilled under the fairly mild assumption that $\operatorname{ker} S(\varphi)$ is not fully contained in $\{z: \operatorname{det}(R(z))=0\}$, and by selecting $\epsilon>0$ and $K>0$ sufficiently small and sufficiently large, respectively. In fact, one can observe that the $\lambda$-level set of $F(z)$, i.e. $\mathcal{E}_{\lambda}=\{z: F(z)=\lambda\}$ for some $\lambda \geq 0$, coincides with the set $\left\{z: z^{T}(S(\varphi)-\lambda I) z=0\right\}$ which is a convex cone, and then, invoking a space separation principle, at least one direction $z_{i}$ can be found such that the gradient of $F(z)$ along such direction points toward a level set $\mathcal{E}_{\lambda}$ with $F(z)>\lambda$. Moreover, if the feasible set is made sufficiently large by a suitable choice of the constants $\epsilon, K$, a point $z^{+}$can always be intercepted on the lower level set $\mathcal{E}_{\lambda}$ without violating the desired constraint $\operatorname{det}\left(R\left(z^{+}\right)\right) \neq 0$.

Remark 2: We point out that the proposed algorithm is only one possible scheme among many others. However, one key advantage of the proposed choice is the possibility to recast the nonlinear constraint $\operatorname{det}\left(R\left(z^{+}\right)\right) \neq 0$ into a finite bank of scalar convex constraints by means of the family of disjoint sets $\Lambda(\epsilon, \bar{z}, i), i=1, \ldots, p$, this allowing to implement a computationally efficient procedure for searching the optimal weights $z$. 


\section{EXAMPLE 1 REVISITED}

Example 2: Consider again the linear plant of Example 1 under the same operative conditions. We point out that the plant is output redundant, i.e. the given output matrix $C$ corresponds to a configuration where a pair of identical sensors is available. Using the estimated bias $\hat{\varphi}_{k}$ obtained in Example 1, an observer has been designed according to (13) where the update map $G$ has been shaped based on Algorithm 2 and Proposition 3. The tunable parameters in the algorithm have been selected as $\epsilon=0.01$, $K=10$. The behavior of the estimation error is depicted in Figure VI (top), where the asymptotic settling to zero is appreciable. Moreover, in Figure VI (bottom), the convergence of dynamic weights to the optimal values is illustrated.

$$
\begin{gathered}
\text { In particular one has } z_{k} \rightarrow z_{\text {opt }}=\left[\begin{array}{r}
2 / \sqrt{5} \\
-1 / \sqrt{5}
\end{array}\right] \text { and the optimal pseudoinverse is } \\
\Pi\left(z_{\text {opt }}\right)=\left[Y^{T} \operatorname{diag}\left(z_{\text {opt }}\right) Y\right]^{-1} Y^{T} \operatorname{diag}\left(z_{\text {opt }}\right)=\left[\begin{array}{ll}
2 & -1
\end{array}\right]
\end{gathered}
$$

with $\Pi\left(z_{\text {opt }}\right) \varphi=0$.

The perturbed case (7) has also been tackled with the output allocation scheme. To prevent a noisy estimation response, the scaling and filtering algorithm proposed in Section IV-B has been implemented to generate a filtered estimate $\hat{\varphi}_{k}^{* *}$, whose high performances are depicted in Figure VI (top) and compared to those of the observer with direct estimated bias injection discussed in Example 1. As clearly visible in the figure, the effect of the bias is attenuated in the estimation error $\left|x_{k}-\bar{x}_{k}\right|$ when the estimated bias is directly injected, while is almost fully compensated in the estimation error $\left|x_{k}-\hat{x}_{k}\right|$ when output allocation is performed. Finally, the convergences of weights $z_{k}^{* *}$ to the optimal values is shown in Figure VI (bottom). It is worth to note that, due to noise, the evaluated optimal weights are opposite to those obtained in the noise-free scenario. However, this fact does not provide any change in terms of performances since the resulting optimal pseudo-inverse is the same.

\section{CONCLUSIONS}

A novel robust estimation scheme has been proposed based on a pseudoinverse with adaptive weights when designing linear Luenberger observers in the presence of redundant measurements for discretetime linear plants. The paper extends to the discrete-time setting some results originally obtained by the authors in the case of continuous-time systems [11]. The proposed architecture, which combines two different observers with interconnected purposes, is successful at rejecting constant biases affecting the sensor measurements. The first observer is in charge of estimating the bias, and the second estimator uses dynamic pseudoinverses to compute the best combination of sensors. One key advantage of the method is that it does not require any knowledge on the structure of the perturbations, i.e. no matching condition is involved. Moreover, the robustness of estimation performances with respect to measurement noise is a desirable additional feature of the proposed approach.

\section{REFERENCES}

[1] T. A. Johansen and T. I. Fossen, "Control allocation: A survey," Automatica, p. in press, 2013.

[2] L. Zaccarian, "Dynamic allocation for input redundant control systems," Automatica, vol. 45, pp. 1431-1438, 2009.

[3] D. Catlin, Estimation, control, and the discrete Kalman filter. Springer Science \& Business Media, 2012, vol. 71.

[4] R. H. Rogne, T. A. Johansen, and T. I. Fossen, "Observer and imu-based detection and isolation of faults in position reference systems and gyrocompasses with dual redundancy in dynamic positioning," in Control Applications (CCA), IEEE Conference on, 2014, pp. 83-88.

[5] I. Samy, I. Postlethwaite, and D. W. Gu, "Survey and application of sensor fault detection and isolation schemes," Control Engineering Practice, vol. 19, no. 7, pp. 658-674, 2011.

[6] C. Commault, J. M. Dion, O. Sename, and R. Motyeian, "Observer-based fault detection and isolation for structured systems," IEEE Trans. on Automatic Control, vol. 47, no. 12, pp. 2074-2079, 2002.

[7] A. Pertew, J. Marquez, H, and Q. Zhao, "LMI-based sensor fault diagnosis for nonlinear lipschitz systems," Automatica, vol. 43, no. 8, pp. 1464-1469, 2007.

[8] E. Franco, R. Olfati-Saber, T. Parisini, and M. M. Polycarpou, "Distributed fault diagnosis using sensor networks and consensus-based filters," in IEEE Conf. Decision and Control, 2006, pp. 386-391. 

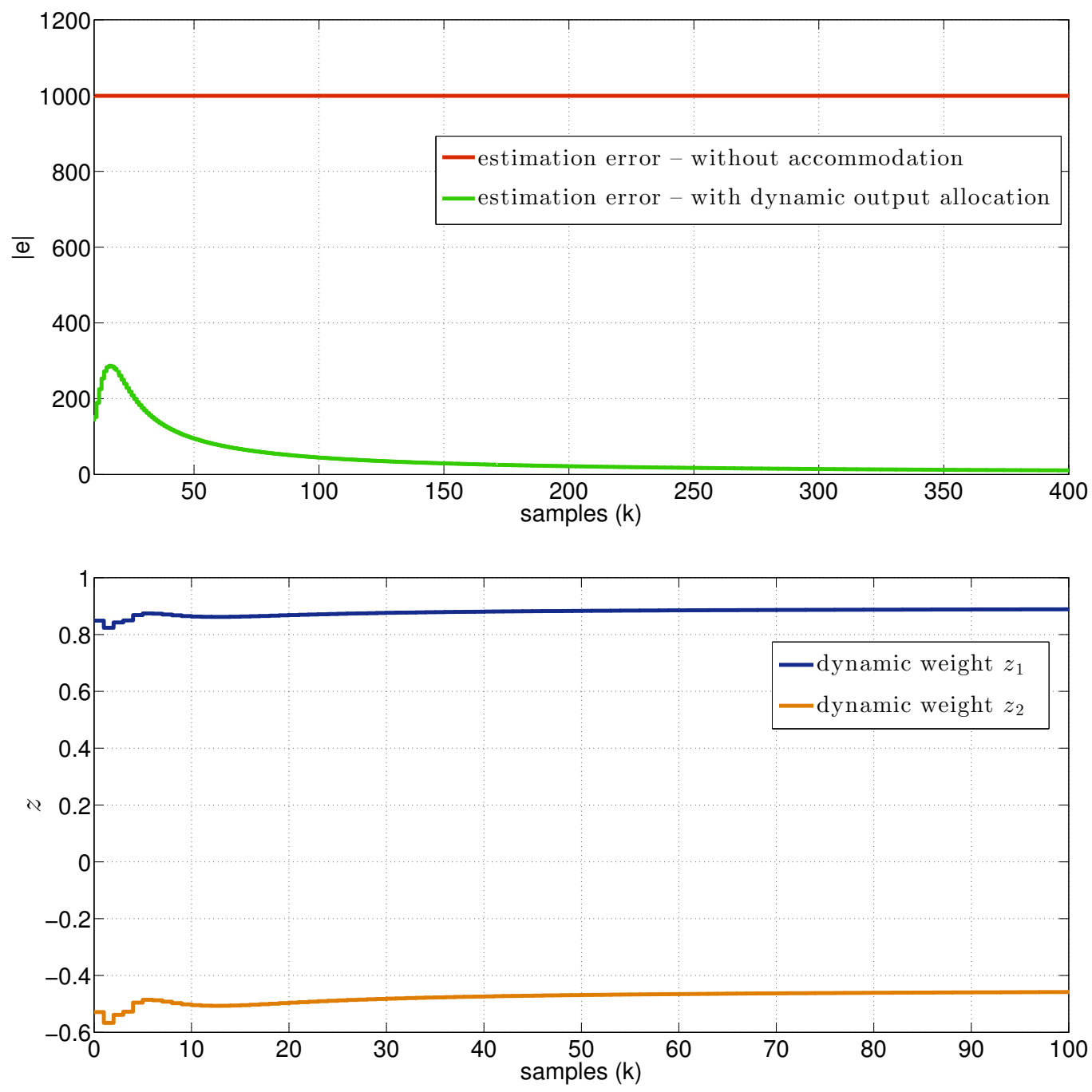

Fig. 3. Example 2: Estimation errors (top) and dynamic weights (bottom) without noise.

[9] V. Reppa, M. M. Polycarpou, and C. G. Panayiotou, "Adaptive approximation for multiple sensor fault detection and isolation of nonlinear uncertain systems," IEEE Trans. on Neural Networks and Learning Systems, vol. 25, no. 1, pp. 137-153, 2014.

[10] R. Luo, S. Misra, M.and Qin, R. Barton, and D. Himmelblau, "Sensor fault detection via multiscale analysis and nonparametric statistical inference," Industrial \& Engineering Chem. Res., vol. 37, no. 3, pp. 1024-1032, 1998.

[11] A. Cristofaro and L. Zaccarian, "An adaptive pseudo-inverse method for the fault-tolerant output allocation in linear observers with redundant sensors," IEEE Conf. on Decision and Control, pp. 5402-5407, 2016.

[12] P. Seibert and J. Florio, "On the reduction to a subspace of stability properties of systems in metric spaces," Annali di Matematica pura ed applicata, vol. 169, no. 1, pp. 291-320, 1995.

[13] J. Sherman and W. J. Morrison, "Adjustment of an inverse matrix corresponding to a change in one element of a given matrix," The Annals of Mathematical Statistics, vol. 21, no. 1, pp. 124-127, 1950. 

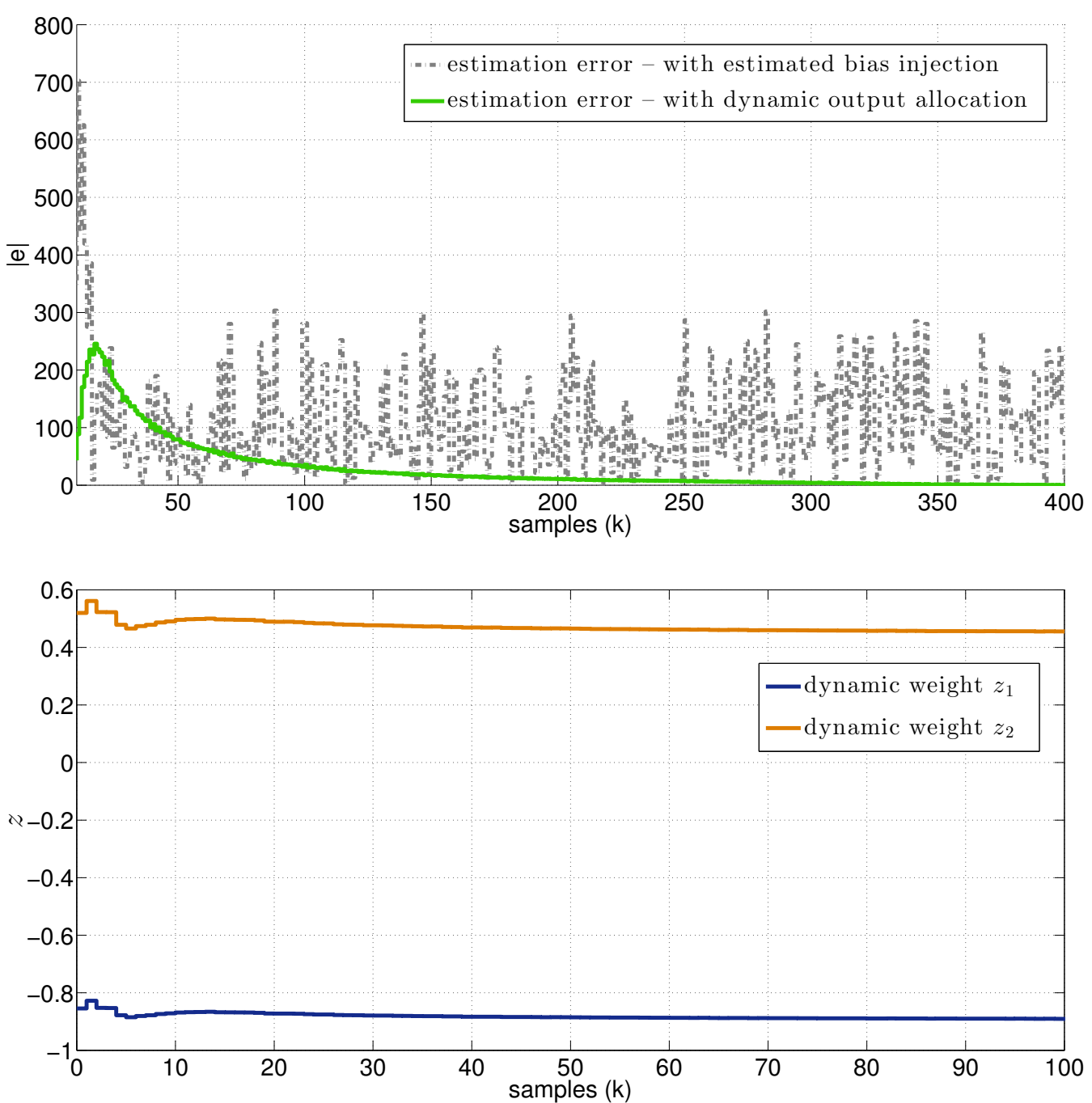

Fig. 4. Example 2: Estimation errors (top) and dynamic weights (bottom) with noise. 